УДК 519.6

\author{
Н. Н. Беляев *, И. В. Калашников**, Е.Ю. Гунько ${ }^{*}$, О.П. Савина* \\ *Днепропетровский национальный университет железнодорожного транспорта \\ им. академика В. Лазаряна \\ **Государственное предприятие «Проектно-изыскательский институт \\ железнодорожного транспорта Украины «Укрзалізничпроект»
}

\title{
3D МОДЕЛЬ ДЛЯ ОЦЕНКИ ТЕРРИТОРИАЛЬНОГО РИСКА
}

Предложена 3-D численная модель для оценки территориального риска при эмиссии химически опасных веществ при авариях на промышленных объектах, транспорте или при теракте с применением химического агента. Эмиссия химического агента происходит возле зданий. Ставится задача разработки модели для оценки территориального риска с учетом различных метеорологических ситуаций и с учетом инфильтрации опасного вещества внутрь зданий. Разработанная численная модель основывается на моделировании процесса рассеивания опасного вещества в атмосфере при различных метеоусловиях, вероятность реализации которых известна. Решение задачи по оценке территориального риска основывается на последовательном решении двух задач. Первая задача - это расчет поля скорости ветрового потока в условиях застройки и при различных метеоситуациях. Данная задача решается с учетом размешения зданий в расчетной области. Для решения этой задачи используется модель потенциального течения. Используемая модель дает возможность быстро, на компьютерах малой и средней мощности, рассчитывать деформацию поля скорости ветрового потока при обтекании зданий. Для численного решения уравнения Лапласа для потенциала скорости используется метод Ричардсона. Вторая задача - это задача о расчете рассеивания опасного вещества в атмосфере при известном поле скорости ветрового потока. Для решения второй задачи используется трехмерная модель массопереноса примеси в атмосфере. Для численного интегрирования уравнения массопереноса используется неявная разностная схема расщепления. Рассмотрена математическая модель для экспресс-расчета динамики загрязнения воздуха внутри помещений. Приведено описание алгоритма решения задачи по оценке территориального риска при эмиссии химически опасного вещества. Представлены результаты вычислительного эксперимента.

Ключевые слова: территориальный риск, теракт, распространение загрязнений, численное моделирование.

Запропоновано 3-D чисельну модель для оцінки територіального ризику при емісії хімічно небезпечних речовин при аваріях на промислових об'єктах, транспорті або під час теракту із застосуванням хімічного агента. Емісія хімічного агента відбувається біля будівель. Ставиться задача розробки моделі для оцінки територіального ризику 3 урахуванням різних метеорологічних ситуацій i 3 урахуванням інфільтрації небезпечної речовини всередину будівель. Розроблена чисельна модель грунтусться на моделюванні процесу розсіювання небезпечної речовини в атмосфері при різних метеоумовах, ймовірність реалізації яких відома. Розв'язок задачі по оцінці територіального ризику грунтусться на послідовному розв'язанні двох задач. Перша задача - це розрахунок поля швидкості вітрового потоку в умовах забудови i при різних метеоситуація. Дана задача вирішується 3 урахуванням розміщення будівель в розрахунковій області. Для розв'язування цієї задачі використовусться модель потенційної течії. Використовувана модель дас можливість швидко, на комп'ютерах малої і середньої потужності, розраховувати деформацію поля швидкості вітрового потоку при обтіканні будівель. Для чисельного розв'язку рівняння Лапласа для потенціалу швидкості використовусться метод Річардсона. Друга задача - це задача про розрахунок розсіювання небезпечної речовини в атмосфері при відомому полі швидкості вітрового потоку. Для розв'язання другої задачі використовується тривимірна модель масопереносу домішки в атмосфері. Для чисельного інтегрування рівняння масопереносу використовусться неявна різницева схема розщеплення. Розглянуто математичну модель для експрес-розрахунку динаміки забруднення повітря

() Беляев Н. Н., Калашников И. В., Гунько Е. Ю., Савина О. П., 2018 90 
всередині приміщень. Наведено опис алгоритму розв'язання задачі по оцінці територіального ризику при емісії хімічно небезпечної речовини. Представлено результати обчислювального експерименту.

Ключові слова: територіальний ризик, теракт, поширення забруднень, чисельне моделювання.

3-D numerical model is proposed for estimating the territorial risk in the emission of chemically hazardous substances in case of accidents at industrial facilities, transport or in a terrorist attack using a chemical agent. Emission of a chemical agent occurs near buildings. The task is to develop a model for assessing territorial risk taking into account various meteorological situations and taking into account the infiltration of hazardous substances into buildings. The developed model model is based on the modeling of the process of dispersal of a hazardous substance in the atmosphere under various meteorological conditions, the probability of which is known. The solution of the task of assessing territorial risk is based on a consistent solution of two tasks. The first task is the calculation of the velocity field of the wind flow in the conditions of building and under various meteorological situations. This task is solved taking into account the location of buildings in the calculation area. To solve this problem, the potential flow model is used. To solve this problem, the potential flow model is used. The model used makes it possible to quickly calculate, on small and medium-power computers, the deformation of the velocity field of the wind flow during flow of buildings. For the numerical solution of the Laplace equation for the velocity potential, the Richardson method is used. The second task is the problem of calculating the dispersion of a hazardous substance in the atmosphere with a known field of wind speed. To solve the second problem, a three-dimensional model of mass transfer of an impurity in the atmosphere is used. For the numerical integration of the mass transfer equation, an implicit difference splitting scheme is used. A mathematical model for the express calculation of the dynamics of indoor air pollution is considered. A description of the algorithm for solving the problem of assessing the territorial risk in the emission of a chemically hazardous substance is given. The results of a numerical experiment are presented.

Key words: territorial risk, terrorist attack, the spread of pollution, numerical simulation.

Актуальность. К числу особо важных задач в области промышленной безопасности относится задача прогноза последствий аварийной эмиссии химически опасных веществ на промышленных объектах, транспорте. К этому классу задач также относится задача оценки риска при терактах, сопровождающихся выбросом химически опасных агентов $[2,3,5,6]$. На практике, для решения таких задач, широкое распространение получили упрощенные модели прогноза, основанные на эмпирических формулах. К этой группе моделей относится методика типа ОНД-86, методика прогноза последствий аварий на химически опасных объектах и транспорте. Другой класс моделей, широко используемых на практике для решения указанных задач, являются Гауссовы модели. Данные модели реализованы в виде специализированных программ, типа ALOHA. Модели данного класса позволяют оперативно получать прогнозные данные, вполне удовлетворяющие практиков. Но перечисленные модели не дают возможности оценивать последствия эмиссии опасных веществ при наличии застройки.

В этой связи актуальной задачей является разработка эффективных методов оценки риска при эмиссии химически опасных веществ в условиях застройки.

Постановка задачи. Рассматривается выброс химически опасного вещества возле зданий (рис. 1). Примером такого выброса может быть аварийная эмиссия на пункте заправки цистерн, или атака террориста в селитебной зоне. Ставится задача оценки территориального риска при такой эмиссии. Известно, что территориальный риск является показателем вероятности поражения людей на определенной территории.

Цель. Ставится задача по разработке 3D численной модели для оценки территориального риска при выбросе химически опасных веществ в условиях застройки. 


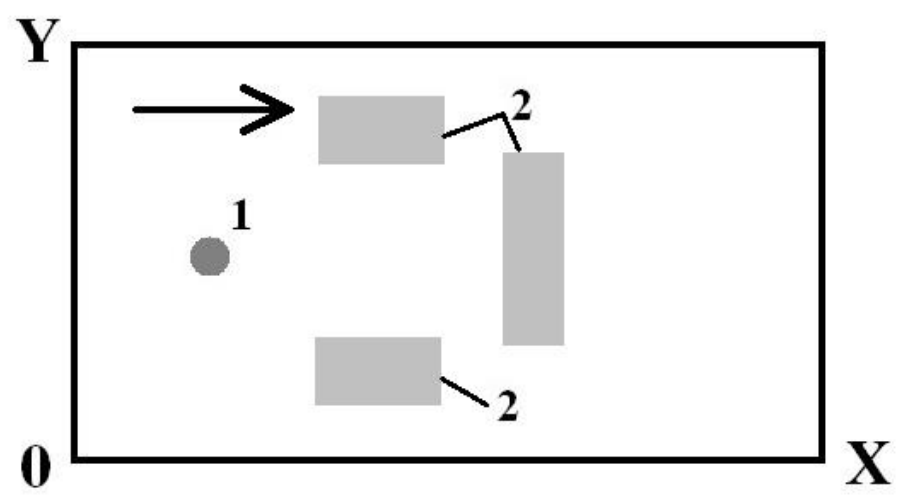

Рис. 1. Расчетная схема:

1 - место эмиссии химически опасного вещества при теракте, 2 - здание

Математическая модель. Оценка территориального риска будет осуществляться в случае выброса химически опасного вещества при наличии зданий. В этой связи возникает задача расчета поля скорости воздушного потока при обтекании зданий. Для решения этой задачи будем использовать модель потенциального течения

$$
\frac{\partial^{2} P}{\partial x^{2}}+\frac{\partial^{2} P}{\partial y^{2}}+\frac{\partial^{2} P}{\partial z^{2}}=0,
$$

где $P$ - потенциал скорости.

Компоненты вектора скорости ветрового потока потока определяются соотношениями

$$
u=\frac{\partial P}{\partial x}, v=\frac{\partial P}{\partial y}, w=\frac{\partial P}{\partial z} .
$$

Применение модели потенциального течения дает возможность оперативно рассчитывать поле скорости воздушного потока при обтекании зданий. Это является важным фактором при использовании на практике прогнозных моделей, когда проводятся серийные расчеты. Известно, что, например, применение LES модели для расчета поля скорости в условиях застройки требует десятки часов на расчет одного варианта задачи, что является серьезным препятствием при использовании ее на практике.

Для моделирования процесса распространения опасных веществ в атмосфере будем использовать трехмерное уравнение переноса примеси [4]:

$$
\begin{aligned}
& \frac{\partial C}{\partial t}+\frac{\partial u C}{\partial x}+\frac{\partial v C}{\partial y}+\frac{\partial\left(w-w_{S}\right) C}{\partial z}+\sigma C= \\
= & \frac{\partial}{\partial x}\left(\mu_{x} \frac{\partial C}{\partial x}\right)+\frac{\partial}{\partial y}\left(\mu_{y} \frac{\partial C}{\partial y}\right)+\frac{\partial}{\partial z}\left(\mu_{z} \frac{\partial C}{\partial z}\right)+ \\
& +\sum Q_{i}(t) \delta\left(x-x_{i}\right) \delta\left(y-y_{i}\right) \delta\left(z-z_{i}\right),
\end{aligned}
$$

где $C$ - концентрация химически опасного вещества в атмосфере ; $u, v, w-$ компоненты вектора скорости ветрового потока; $w_{S}$ - скорость гравитационного 
оседания загрязнителя; $\sigma$ - коэффициент, учитывающий распад загрязнителя; $\mu=\left(\mu_{x}, \mu_{y}, \mu_{z}\right)$ - коэффициенты атмосферной турбулентной диффузии; $Q-$ интенсивность эмиссии опасного вещества $\delta\left(x-x_{i}\right)\left(y-y_{i}\right)\left(z-z_{i}\right)$ - дельта-функция Дирака; $x_{i}, y_{i}, z_{i}$ - координаты источника эмиссии агента; $t$ - время.

Постановка краевых условий для моделирующих уравнений (1), (3) рассмотрена в [2-4].

Для численного интегрирования уравнения (1) используется метод Ричардсона, а для численного интегрирования уравнения переноса примеси применяется неявная разностная схема расщепления [2 - 4].

Изменение концентрации опасного вещества в помещениях при инфильтрации в них загрязненного атмосферного воздуха будем рассчитывать на базе уравнения [3]

$$
\frac{d(C V)}{d t}=Q_{f} C_{\text {out }}-Q_{f} C,
$$

где $C$ - концентрация опасного вещества в выходящем из помещения воздухе; $V$ - объем комнаты; $Q_{f}$ - интенсивность воздухообмена (инфильтрации); $C_{o u t}-$ концентрация опасного вещества в приточном воздухе.

Величина инфильтрации в помещение может быть рассчитана по следующей эмпирической модели [2]

$$
Q=E L A \cdot \sqrt{f_{s}^{2}|\Delta T|+f_{w}^{2} U^{2}},
$$

где $f_{s}$ - параметр, учитывающий процесс инфильтрации через потолок и пол; $f_{w}$ - параметр, учитывающий наличие возле здания иных объектов; ELA - площадь инфильтрации; $\Delta T$ - перепад температур между температурой внутри помещения и снаружи; $U$ - скорость ветра.

Применение уравнения (4) позволяет прогнозировать темп роста уровня загрязнения внутри помещения, а значит определить время, когда будет достигнута максимальная концентрация и будет ли риск поражения людей внутри помещения.

При оценке территориального риска на открытой местности или в помещениях принимаем во внимание, что зона поражения - различна для различных метеорологических ситуаций $P\left(W_{i}\right)$. При построении модели под конкретной метеорологической ситуацией будем понимать конкретное значение скорости и направления ветра. Вероятность реализации конкретной метеоситуации для данного региона определяется на основе статистических данных (или на основе метеопрогноза) [1]:

$$
P\left(W_{i}\right)=N_{\Pi} / T
$$

где $N_{\Pi}$ - число дней (часов), соответствующих конкретной метеорологической ситуации; $T$ - период наблюдений.

Вероятность попадания человека в зону поражения (как на открытой местности так и внутри помещений) определится следующим образом:

$$
P(W)_{\Sigma}=\sum_{i=0}^{n} P\left(W_{i}\right),
$$

где $P(W)_{\sum}$ - суммарная вероятность всех рассматриваемых метеорологических ситуаций, при которых человек находится в зоне, где концентрация опасного 
вещества превышает некоторое пороговое значение, при котором возникает поражение определенной тяжести.

Для расчета вероятности оказаться в зоне токсичного поражения при эмиссии опасного вещества необходимо, для конкретной точки расчетной области, выполнить расчеты по формуле (6). Расчеты выполняются, как для открытого пространства, так и для конкретных помещений. Интенсивность инфильтрации в помещение, концентрация опасного вещества в инфильтрующемся воздухе - также будут различны при различных (вероятных) метеоситуациях. Кроме этого, на эти величины будет влиять место воздухозабора (инфильтрации) на здании, так как в различных местах здания скорость ветрового потока будет различна.

Данная методология решения здачи по оценке террториального риска реализована в виде компьютерной программы «RISK-3». Разработанная модель ориентирована на решение задачи по оценке территориального риска в масштабе «local».

Результаты. Пример практического применения разработанной численной модели показан ниже. На рис. 1 представлена расчетная схема. Рассматривается выброс хлора. Длительность эмиссии - 3 мин. Для региона вероятные значения скорости ветра составляют: 1,5 м/с (вероятность 70\%) и 3,5 м/с (вероятность 30\%).

На рис. 2 представлена зона загрязнения через минуту после начала эмиссии хлора. Направление ветра показано стрелкой. Видно, что возле зданий формируется обширная зона загрязнения, которая охватывает второе здание со всех сторон.

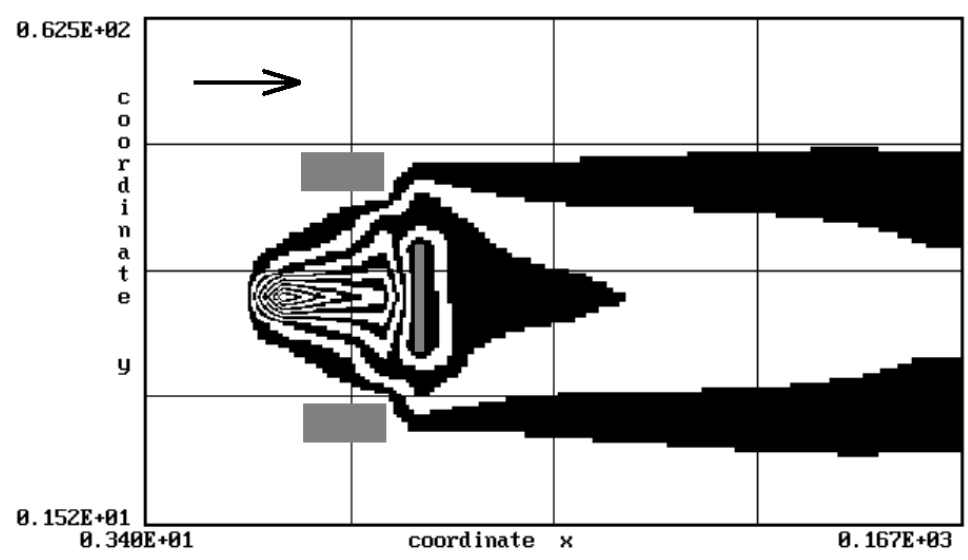

Рис. 2. Зона загрязнения возле зданий при значениях скорости ветра $3,5 \mathrm{~m} / \mathrm{c}, t=62 \mathrm{c}, \mathrm{z}=4 \mathrm{м}$

На рис. 3 представлена матрица территориального риска для рассматриваемого сценария. 


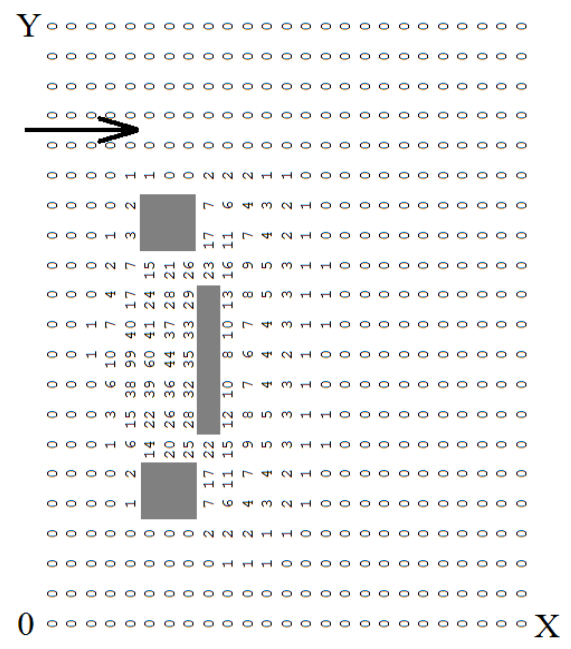

Рис.3. Матрица территориального риска при значениях $t=62 \mathrm{c}, \mathrm{z}=4 \mathrm{м}$

Как видно из представленного рисунка, за вторым зданием риск поражения составляет порядка $10 \%$ - 13\%, то есть здание выполняет определенную защитную функцию.

Выводы. В работе рассмотрена модель оценки территориального риска при эмиссии химически опасных веществ. Основу модели составляет расчет процесса загрязнения атмосферного воздуха в условиях застройки с последующей оценкой размеров зон, попадающих под влияние источника эмиссии химически опасного вещества. Дальнейшее совершенствование данного направления следует проводить в направлении создания 3D модели для расчета территориального риска в масштабе «urban».

\section{Библиографические ссылки}

1. Алымов, В. Т. Техногенный риск: Анализ и оценка: Учебное пособие для вузов [Текст] / В. Т. Алымов, Н. П. Тарасова. - М. : ИКЦ «Академкнига», 2004. - 118 с.

2. Беляев, Н. Н. Защита зданий от проникновения в них опасных веществ: Монография [Текст] / Н. Н. Беляев, Е. Ю. Гунько, Н. В. Росточило. - Д. : «Акцент ПП», 2014. - 136 с.

3. Оценка техногенного риска при эмиссии опасных веществ на железнодорожном транспорте [Текст] / Н. Н. Беляев, Е. Ю. Гунько, П. С. Кириченко, Л. Я. Мунтян. - Кривой Рог : Изд. Р. А. Козлов, 2017. - 127 с.

4. Численное моделирование распространения загрязнения в окружающей среде [Текст] / М. З. Згуровский, В. В. Скопецкий, В. К. Хрущ, Н. Н. Беляев. - К. : Наук. думка, 1997. - 368 c.

5. Biliaiev, M. Numerical Simulation of Indoor Air Pollution and Atmosphere Pollution for Regions Having Complex Topography [Text]/ M. Biliaiev // Air Pollution Modeling and its Application XXI, Springer, 2012. - P. 87-91.

6. Guidance on safety Risk Assessment for Chemical Transport Operations // The European Chemical Industry Council. Cefic. October 2013. - Режим доступа: http:// www.cefic.org, свободный. 